Research Article

\title{
A Simulation Method Based on the Candidate Operation Strategies for the Combined Cooling, Heat, and Power (CCHP) System
}

\author{
Feng Zhu $\mathbb{D}^{\mathbb{D}},{ }^{1}$ Jing-Qi Fu, ${ }^{1}$ Bin Zou, ${ }^{1}$ and Wen-Rong Si $\mathbb{C}^{2}$ \\ ${ }^{1}$ School of Mechatronic Engineering and Automation, Shanghai University, Shanghai 200444, China \\ ${ }^{2}$ State Grid Shanghai Electrical Power Research Institute, Shanghai 200437, China \\ Correspondence should be addressed to Feng Zhu; tsingfeng@shu.edu.cn
}

Received 28 August 2020; Revised 3 February 2021; Accepted 20 February 2021; Published 28 February 2021

Academic Editor: Omar-Jacobo Santos

Copyright (c) 2021 Feng Zhu et al. This is an open access article distributed under the Creative Commons Attribution License, which permits unrestricted use, distribution, and reproduction in any medium, provided the original work is properly cited.

With the change of the distributed Combined cooling, heat, and power (CCHP) system operation environment, such as the operation under the power market, CCHP needs to adopt the operation strategy based on cost minimization instead of the traditional following thermal load strategy (FTL). However, the current CCHP system simulation adopts FTL or FEL or a combination of the two; whether these strategies have the least cost has not been confirmed. In this paper, all kinds of operation strategies of the CCHP with two cooling methods are investigated according to the KKT conditions (Karush-Kuhn-Tucker conditions) of the optimal operation model. Among all the feasible operation strategies, most of them do not meet the KKT condition, and only 43 strategies may be the optimal ones that constitute candidate set. The conditions of the optimal operation strategy in the candidate set are obtained, and it is easy to calculate, so the optimal operation strategy can be easily selected from the candidate set. Some previously unnoticed strategies, for example, TEWM, PE-FPG, FTL-R, and FEL-L, may become optimal. A rule-based CCHP simulation method is established that selects the strategy with minimal cost as the optimal strategy from the candidate strategy set. The presented method has small time consumption and can simulate the optimal operation. The case study verified the characteristics of the proposed simulation method.

\section{Introduction}

The distributed combined cooling, heat, and power (CCHP) system has been supported by various governments because of its high primary energy efficiency and low emissions $[1,2]$. The operation simulation of the CCHP as a tool to estimate the operation costs is important to the investment planning of CCHP [3, 4].

The traditional operation strategies in CCHP simulation are the following thermal load (FTL), the following electric load (FEL), and the hybrid electric-thermal load (HET). FTL or FEL is used, respectively, according to load conditions in HET. When the electric load is larger, FTL strategy is adopted; when the thermal load is larger, FEL strategies are adopted $[5,6]$. In reference [6], the quasi-steady models of the combined heating and power (CHP) and CCHP were constructed to simulate the production of CHP, performances of CCHP and CHP systems under FTL or FEL based on primary energy consumption, operation cost, and carbon dioxide emissions for different climate conditions. The simulation method of CHP and CCHP has almost become the standard method to estimate the performance of CHP and CCHP. In reference [7], the performance of FEL operation strategy for CHP with energy storage and large fluctuation of electric load is estimated by the simulation method. Reference [8] estimates the performance characteristics of CCHP system under FTL and FEL strategies by using simulation models of absorption refrigeration, electric refrigeration, natural gas fuel absorption refrigeration, and geothermal refrigeration in CCHP. Using the simulation, literature [9] Using simulation method, literature [9] examined the economic feasibility of the combined cooling, heating, and power (CCHP) systems 
when it was applied in the Department of Energy (DOE) reference large office buildings in three cities of the U.S.: San Francisco, Boston, and Miami. In the above simulation models, the operation strategy of CCHP is FTL or FEL, or one of them is adopted under certain conditions. Comparison of exhaust-gas-and-hot-water-driven absorption chiller (AC) and another three cooling modes, including AC combined with electric chiller (EC), AC combined with gas-fired absorption chiller (GFC), and AC combined with ground source heat pump (GSHP) based on the simulation model, is made in reference [10]. The results show that the CCHP system with GSHP under FEL strategy has the best comprehensive performance.

In addition, some authors have proposed some new strategies and evaluated their performance by simulation. In reference [11], the new operation strategies for the CCHP system with hybrid chillers, consisting of a combined electric and absorption chiller, are investigated by simulation, based on the variational electric cooling to cool load ratio. In $[12,13]$, another new CCHP operation strategy is proposed, and the advantages of the new operation strategy are also illustrated by simulation. In reference [11], a variety of new operation strategies have been developed by combining electric chiller and absorption chiller, which have better performance than the traditional FTL and FEL. However, this paper does not further investigate the optimal strategy. References $[12,13]$ also show by simulation that the new strategy proposed by the authors has better performance than FTL and FEL strategies but also fails to find the optimal strategy. The simulation method can obtain the performance of CCHP system according to the determined operation strategy, such as primary energy utilization rate, operation cost, carbon dioxide emission, and so on. However, it is uncertain whether the commonly used operation strategies, FTL and FEL, are the least cost strategies, which are closely related to the operating environment of the CCHP system, such as cooling and heating load, electricity price, and so on.

The optimal combination of the power and heat sources' outputs to satisfy heat and power demand of system and operational constraints is known as CHP (or CCHP) economic dispatch (CHPED) problem and has attracted a lot of interests, especially in the electricity market environment $[10,13-16]$. The CHPED problem is a complex optimization problem to meet the demand of cooling, heating, and power loads at the same time. The optimal operation problem is modeled as a nonconvex nonlinear programming and was solved by the branch and bound method [17], the Lagrange relaxation method [18], and particle swarm algorithm [19]. These operating models, however, are too time-consuming while they were used as a tool to estimate the annual operation cost of the long-term investment problem, so the nonconvex optimal operation model usually was simplified as linear programming or mixed linear integer programming while the generation efficiency was assumed as a constant $[20,21]$. Even so, the computation time is still unacceptable when the annual operation cost is estimated using the linear programming by hourly rolling calculation in CCHP planning problems. Therefore, some typical scenarios are selected to represent the whole year for reducing the computation time, which is called the multiscenario method [22].

In the present paper, the optimal operation strategy of CCHP is tested based on the KKT condition of the optimal operation model of CCHP, and the candidate optimal operation strategy set is obtained. Based on the set, a new simulation method of the CCHP is proposed, which can describe the optimal operation.

The main contributions of this paper are as follows:

(1) Based on the output position of generating units, this paper defines all operation strategies of CCHP system and gives the conditions for each strategy to be the optimal strategy. KKT condition analysis based on the optimal operation model shows that when all cooling modes work simultaneously, and only TEWM, PE-FPG, PE-MPG, BW-FPG, PE-MPG, and BE-MPG are possible optimal, while other combination strategies are not optimal. When the cold demand is met by electric chiller or absorption chiller alone, traditional FTL-L and FEL-R may be the optimal strategies in some cases, but there are also other strategies that may be the optimal strategies, such as FTL-R and FEL-L.

(2) Based on the optimal conditions of CCHP system operation strategy, this paper develops a CCHP operation simulation algorithm. By judging the optimal conditions of CCHP strategy, the algorithm selects the operation strategy with the minimum cost to run and realizes the operation simulation based on the optimal cost. Compared with the conventional simulation algorithms based on FTL or FEL, the algorithm realizes the operation simulation of CCHP according to the minimum cost; compared with the operation simulation based on optimization model, the algorithm has obvious advantages in computing speed because of avoiding iterative optimization. The presented simulation model can be used to estimate the operation cost in the CCHP investment decision making.

This paper is organized as follows. In Section 2, the operation model of CCHP is given. In Section 3, the candidate optimal operation set is obtained. In Section 4, a simulation method based on the optimal operation set is presented. Section 5 presents the case study.

\section{Problem Formulation}

A schematic of the CCHP system is shown in Figure 1. There are three energy buses in the system, namely, heat bus, cold bus, and power bus.

The energy demands of consumers are served for three ways: (1) power is provided by power generation unit (PGU) or/and power grids; (2) heat is provided by waste heat recovery from PGU or/and auxiliary boiler; (3) cooling is provided by electric chiller or/and absorption chiller.

The objective function of the operation problem is minimizing the operation cost. The optimal operation model can be expressed as 


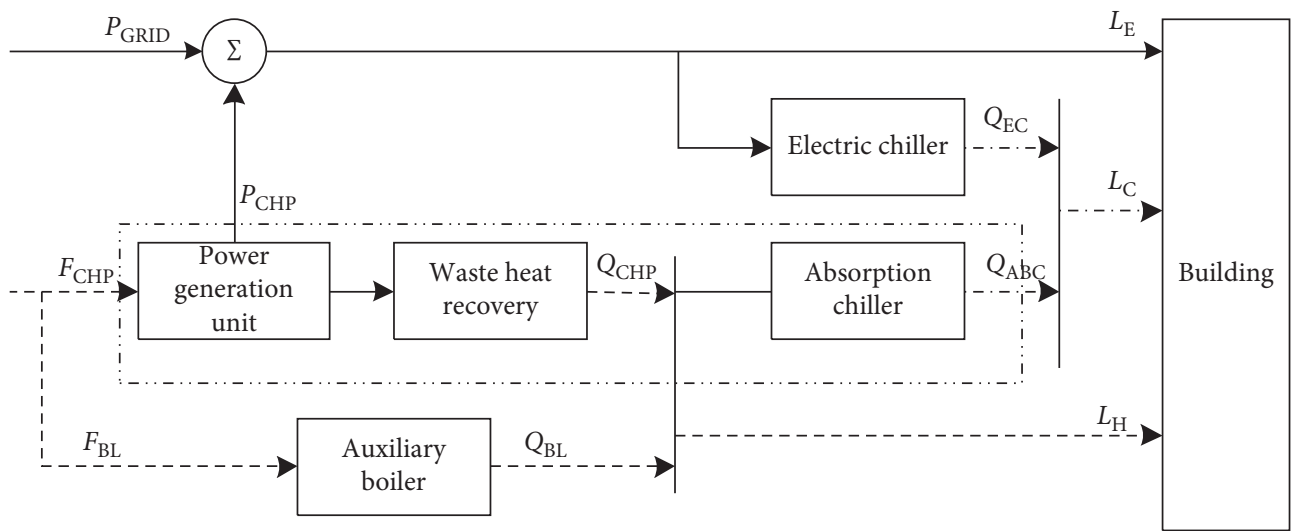

Figure 1: Schematic of the CCHP system.

$$
\begin{aligned}
& \min \left(\frac{\rho_{\mathrm{CHP}}}{\mathrm{LHV}}\right) \cdot F_{\mathrm{CHP}}(t)+\left(\frac{\rho_{\mathrm{BL}}}{\mathrm{LHV}}\right) \cdot F_{\mathrm{BL}}(t)+x \cdot \rho_{\mathrm{GRID}}^{\text {Purchase }}(t) \cdot P_{\mathrm{GRID}}^{\text {Purchase }}(t)-(1-x) \rho_{\mathrm{GRID}}^{\text {Sell }}(t) \cdot P_{\mathrm{GRID}}^{\mathrm{Sell}}(t), \\
& L_{E}(t)-P_{\mathrm{CHP}}(t)-x \cdot P_{\mathrm{GRID}}^{\text {Purchase }}(t)+(1-x) \cdot P_{\mathrm{GRID}}^{\mathrm{Sell}}+\frac{\mathrm{Q}_{\mathrm{EC}}(t)}{\mathrm{COP}_{\mathrm{EC}}}=0 \text {, } \\
& L_{H}(t)+\frac{Q_{\mathrm{ABC}}(t)}{\mathrm{COP}_{\mathrm{ABC}}}-Q_{\mathrm{CHP}}(t)-Q_{\mathrm{BL}}(t) \leq 0, \\
& L_{C}(t)-Q_{\mathrm{ABC}}(t)-Q_{\mathrm{EC}}(t)=0, \\
& P_{\mathrm{CHP}}(t)=F_{\mathrm{CHP}}(t) \cdot \eta_{\mathrm{CHP}, E}, \\
& Q_{\mathrm{CHP}}(t)=F_{\mathrm{CHP}}(t)\left(1-\eta_{\mathrm{CHP}, E}\right) \cdot \eta_{\mathrm{CHP}, H} \\
& f=\frac{P_{\mathrm{CHP}}}{P_{\mathrm{CHP}, \mathrm{MAX}}}, \\
& \eta_{\mathrm{CHP}, \mathrm{E}}=a+b \cdot f+c \cdot f^{2}, \\
& \mathrm{Q}_{\mathrm{BL}}(t)=F_{\mathrm{BL}}(t) \cdot \eta_{\mathrm{BL}}, \\
& P_{\mathrm{CHP}, \mathrm{MIN}} \leq P_{\mathrm{CHP}}(t) \leq P_{\mathrm{CHP}, \mathrm{MAX}} \\
& Q_{\mathrm{BL}, \mathrm{MIN}} \leq Q_{\mathrm{BL}}(t) \leq Q_{\mathrm{BL}, \mathrm{MAX}} \\
& Q_{\mathrm{EC}, \mathrm{MIN}} \leq \mathrm{Q}_{\mathrm{EC}}(t) \leq Q_{\mathrm{EC}, \mathrm{MAX}}, \\
& Q_{\mathrm{ABC}, \mathrm{MIN}} \leq Q_{\mathrm{ABC}}(t) \leq Q_{\mathrm{ABC}, \mathrm{MAX}}, \\
& x \cdot P_{\mathrm{GRID}, \mathrm{MIN}}^{\mathrm{Purchase}} \leq x \cdot P_{\mathrm{GRID}}^{\text {Purchase }}(t) \geq x \cdot P_{\mathrm{GRID}, \mathrm{MAX}}^{\text {Purchase }}, \\
& (1-x) \cdot P_{\mathrm{GRID}, \mathrm{MIN}}^{\text {Purchase }} \leq(1-x) \cdot P_{\mathrm{GRID}}^{\text {Purchase }}(t) \geq(1-x) \cdot P_{\mathrm{GRID}, \mathrm{MAX}}^{\text {Purchase }} \\
& x \in\{0,1\} \text {. }
\end{aligned}
$$


The decision variables of the operation problem are the output of devices and the purchasing/selling electricity energy at times $t$, including $P_{\mathrm{CHP}}(t), Q_{\mathrm{ABC}}(t), \mathrm{Q}_{\mathrm{EC}}(t)$, $Q_{\mathrm{BL}}(t), P_{\mathrm{GRID}}^{\text {Purchase }}(t), P_{\mathrm{GRID}}^{\mathrm{Sell}}(t), t=1, \ldots \ldots, 8760$. Purchasing electricity from grids and selling electricity to grids cannot happen at the same time. The $0-1$ variable $x=1$ expresses purchasing electricity, and $x=0$ expresses selling electricity.

Equations (2)-(4) represent the load balance constraints. Since in some operation strategies, heat is abandoned, the heat for equation (3) is not balanced. Equation (5) is the relationship between PGU's generation and fuel consumption; equation (6) is the relationship between waste heat recovery and PGU's generation; equation (7) defines the PGU's output ratio; equation (8) is the PGU's efficiency function which is the quadratic function of PGU's output ratio. By substituting equation (5) into equation (6), the relationship between the waste heat recovered and power generation can be expressed as equation (17) according to equations (5)-(8), and the $Q-E$ curve in Figure 2 is its geometric representation. Equation (9) is the relationship between boiler heat production and its fuel consumption. Equations (10)-(15) are the limit of devices' output. These expressions of these constraints are the same as those in [11-13], and the $Q-E$ curve is also the same as that in [11-13].

$$
Q_{\mathrm{CHP}}(t)=Q_{\mathrm{Q}-E}\left(P_{\mathrm{CHP}}(t)\right)=\frac{P_{\mathrm{CHP}}(t)\left(1-\eta_{\mathrm{CHP}, E}\right) \cdot \eta_{\mathrm{CHP}, H}}{\eta_{\mathrm{CHP}, E}} .
$$

The above model is a mixed nonlinear integer programming. Because purchasing and selling electricity are not implemented simultaneously, it can be decomposed into two nonlinear programming problems that are called purchasing electricity operation model when $x=1$ and selling electricity operation model when $x=0$, respectively.

\section{The Operation Strategies}

3.1. The Equivalent Load and Cooling Strategies. Assume the cold, heat, and electricity loads are $L_{C}(t), L_{H}(t)$, and $L_{E}(t)$ at time $t$; if the electric chiller supplies the cooling demand, the equivalent electricity load can be obtained as equation (18), and the equivalent load profile $\left(L_{H}(t), L_{E, E}(t)\right)$ is called electric cooling equivalent load (ECEL).

$$
L_{E, E}(t)=\frac{L_{E}(t)+Q_{\mathrm{EC}}(t)}{\operatorname{COP}_{\mathrm{EC}}}
$$

If the absorption chiller supplies the cooling demand, the equivalent heat load can be calculated as equation (19). The load profile $\left(L_{H, E}(t), L_{E}(t)\right)$ is called thermal cooling equivalent load (TCEL).

$$
L_{H, E}(t)=\frac{L_{H}(t)+Q_{\mathrm{ABC}}(t)}{\mathrm{COP}_{\mathrm{ABC}}} .
$$

In Figure 2, the equivalent load is a point, for example, the ECEL is $A 1$ and the TCEL is A2. TCEL is always in the lower right of ECEL.
The points in the line connecting the ECEL and TCEL represent the equivalent loads in which the electric chiller and the absorption chiller work simultaneously to supply the cooling service. The line connecting ECEL and TCEL can be represented as

$$
L_{\mathrm{EEL}}(t)=\frac{L_{E, E}-L_{E}(t)}{L_{H}-L_{H, E}} \cdot\left(L_{\mathrm{EHL}}(t)-L_{H, E}\right)+L_{E}(t) .
$$

3.2. The Candidate Optimal Operation Strategies for Electric Cooling or Thermal Cooling. If ECEL meets the following conditions, it is on the left side of the $Q-E$ curve as $A 1$ and $B 1$ in Figure 2 or on the left of the maximal heat recovery as $D 1$.

$$
\begin{cases}L_{H}(t) \leq Q_{Q-E}\left(L_{E, E}(t)\right), & \text { if } L_{E, E}(t) \leq P_{\mathrm{CHP}, \mathrm{MAX}}, \\ L_{H}(t) \leq Q_{\mathrm{CHP}, \mathrm{MAX}}, & \text { if } L_{E, E}(t)>P_{\mathrm{CHP}, \mathrm{MAX}}\end{cases}
$$

There are seven types of operation strategies to meet the ECEL. FTL (following thermal load) and FEL (following electricity load) are the famous strategies. Using FTL, the thermal load is met by the recovered heat, the power generation is less than the electric load, and the electricity energy needs to be purchased from the grid. Like $A 1$ and ECEL in Figure 2, the working point of FTL is WA11 on the Q-E curve. When the FEL is carried out, the power generation equals electricity demand, and heat recovered is greater than the heat load, and the extra heat energy is wasted. For point $A 1$ in Figure 2, the working point of FET is WA12.

IG (insufficient generation) has the characteristics that the generation is smaller than the electricity load and the recovered heat less than the heat load, so it needs to buy the power from the grid and supply heating by boiler. For example, ECEL is the point $A 1$, whose work points using IG are the points on the Q-E curve between MW and WA11.

Abandoning heat and purchasing electricity (AHPE) is other strategy in which the power generation is less than the electric load, and the recovered heat is greater than the heat demand. If ECEL are $A 1$ points, the working points of AHPE are points of the Q-E curve between WA11 and WA12.

Abandoning heat and selling electricity (AHSE) has the characteristics that more electricity is generated than load, the excess electricity is sold to the grid, the recovered heat is larger than the heat load, and the excess heat is wasted. The working points using AHSE for $A 1$ in Figure 2 are the points between WA12 and FL on the Q-E curve.

The TCEL is on the left of the boundary if the following conditions are met.

$$
\begin{cases}L_{H}(t) \leq Q_{Q-E}\left(L_{E}(t)\right), & \text { if } L_{E}(t) \leq P_{\mathrm{CHP}, \mathrm{MAX}} \\ L_{H}(t) \leq Q_{\mathrm{CHP}, \mathrm{MAX}}, & \text { if } L_{E}(t)>P_{\mathrm{CHP}, \mathrm{MAX}}\end{cases}
$$

There are also seven strategies for the TCEL. So, prefix EC- and TC- are used to distinguish ECEL and TCEL, and suffix -L (-R) indicates that the equivalent load is on the left (right) side of the boundary.

If ECEL does not meet equation (21), the ECEL is on the right side of the $Q-E$ curve; as $C 1$ and $E 1$, there are also seven strategies to meet the equivalent load. 


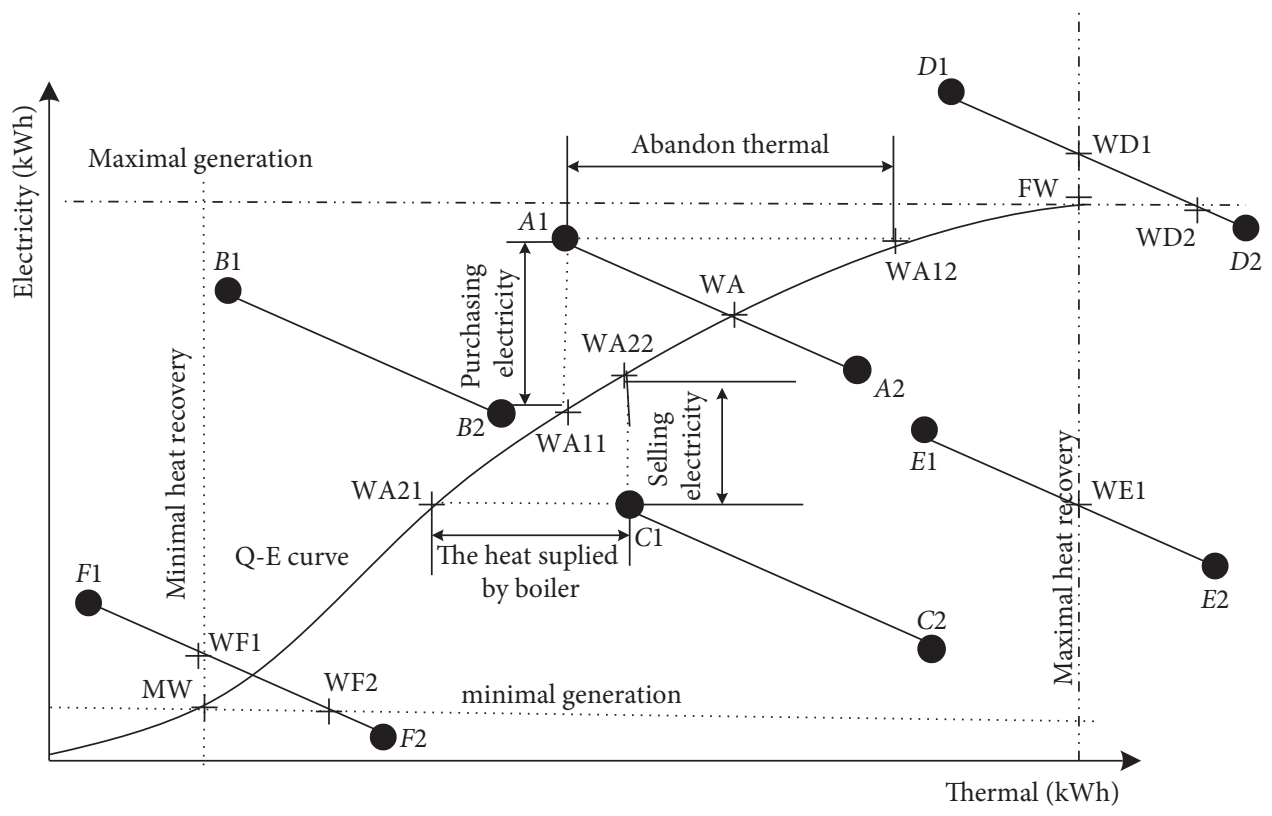

FIgUre 2: The equivalent load and their work points.

EC-MPG-R, EC-FPG-R, and EC-IG-R are the same as EC-MPG-L, EC-FPG-L, and EC-IG-L strategies.

EC-FEL-R is a following electricity load strategy in which the power generation equals electricity load and heat recovered is less than heat load. The boiler must work to supply the thermal. The work point using EC-FEL-R for $C 1$ is the WA21.

EC-FTL-R is a following thermal load strategy in which the power generation is greater than electricity load. The surplus electricity will be sold to the grid, and heat recovered is equal to heat load. For point $\mathrm{C} 2$ in Figure 2, the working point of FET-R is WA22.

EC-BWSE-R has the characteristics that the power generation is greater than the equivalent electricity load, and the recovered heat is less than the heat load. The excess power is sold to the grid, and the lack of heat is supplied by boilers. For the equivalent load point $C 1$, the working points using the BWSE-R are the points on the Q-E curve between WA21 and WA22.

EC-AHSE-R is similar to the EC-AHSE-L strategy. For point $C 1$, the working point is between point WA22 and point FL on the Q-E curve.

If TCEL does not meet equation (22), it is on the right of the boundary. There are the same strategies as ECEL. All 28 kinds of operation strategies are shown in Table 1. The column strategy in Table 1 gives the name of the strategy, while the column $P_{\mathrm{CHP}}(t)$ gives the position of the working point of the strategy. If the strategy corresponds to a certain working point, the output of the PGU is $P_{\mathrm{CHP}}(t)$; otherwise, the output range of the PGU is given. For example, the strategy EC-FTL$\mathrm{L}$ is a fixed operating point where the waste heat recovery is equal to the thermal load $L_{H}(t)$. The corresponding PGU output is $Q^{-1}\left(L_{H}(t)\right.$. And strategy EC-IG-L represents a series of working positions and is represented by the range of its power generation output. $P_{\text {CHP,MIN }}(t)<P_{\text {CHP }}(t)<Q_{Q-E}^{-1}\left(L_{H}(t)\right), Q_{Q-E}^{-1}\left(L_{H}(t)\right)$ is the inverse function of equation (17).
TABLE 1: The operation strategies for ECEL and TCEL.

\begin{tabular}{|c|c|c|}
\hline Strategy & & $P_{\mathrm{CHP}}(t)$ \\
\hline EC-MPG-L & $\sqrt{ }$ & $P_{\mathrm{CHP}, \mathrm{MIN}}$ \\
\hline EC-IG-L & $*$ & $\left(P_{\mathrm{CHP}, \mathrm{MIN}}, Q_{Q-E}^{-1}\left(L_{H}(t)\right)\right.$ \\
\hline EC-FTL-L & $\sqrt{ }$ & $Q_{Q-E}^{-1}\left(L_{H}(t)\right)$ \\
\hline EC-AHPE-L & $x$ & $\left(Q_{Q-E}^{-1}\left(L_{H}(t), L_{E, E}(t)\right)\right.$ \\
\hline EC-FEL-L & $x$ & $L_{E, E}(t)$ \\
\hline EC-AHSE-L & $x$ & $\left(L_{E, E}(t), P_{\text {CHP.MAX }}\right)$ \\
\hline EC-FPG-L & $x$ & $P_{\text {CHP.MAX }}$ \\
\hline TC-MPG-L & $\sqrt{ }$ & $P_{\mathrm{CHP}, \mathrm{MIN}}$ \\
\hline TC-IG-L & $*$ & $\left(P_{\mathrm{CHP}, \mathrm{MIN}}, Q_{Q-E}^{-1}\left(L_{H, E}(t)\right)\right.$ \\
\hline TC-FTL-L & $\sqrt{ }$ & $Q_{Q-E}^{-1}\left(L_{H, E}(t)\right)$ \\
\hline TC-AHPE-L & $*$ & $\left(Q_{Q-E}^{-1}\left(L_{H, E}(t), L_{E}(t)\right)\right.$ \\
\hline TC-FEL-L & $\sqrt{ }$ & $L_{E}(t)$ \\
\hline TC-AHSE-L & $*$ & $\left(L_{E}(t), P_{\text {CHP.MAX }}\right)$ \\
\hline TC-FPG-L & $\sqrt{ }$ & $P_{\text {CHP.MAX }}$ \\
\hline EC-MPG-R & $\sqrt{ }$ & $P_{\mathrm{CHP}, \mathrm{MIN}}$ \\
\hline EC-IG-R & $*$ & $\left(P_{\mathrm{CHP}, \mathrm{MIN}}, L_{E, E}(t)\right)$ \\
\hline EC- FEL - R & $\sqrt{ }$ & $L_{E, E}(t)$ \\
\hline EC- BWSE -R & $*$ & $\left(L_{E, E}(t), Q_{Q-E}^{-1}\left(L_{H}(t)\right)\right)$ \\
\hline EC-FTL-R & $\sqrt{ }$ & $Q_{Q-E}^{-1}\left(L_{H}(t)\right)$ \\
\hline EC-AHSE -R & $x$ & $\left(Q_{Q-E}^{-1}\left(L_{H}(t)\right), P_{\text {CHP.MAX }}\right)$ \\
\hline EC- FPG -R & $x$ & $P_{\text {CHP.MAX }}$ \\
\hline TC-MPG-R & $\sqrt{ }$ & $P_{\mathrm{CHP}, \mathrm{MIN}}$ \\
\hline TC-IG-R & $*$ & $\left(P_{\mathrm{CHP}, \mathrm{MIN}}, L_{E}(t)\right)$ \\
\hline TC-FEL-R & $\sqrt{ }$ & $L_{E}(t)$ \\
\hline TC- BWSE - R & $*$ & $\left(L_{E}(t), Q_{Q-E}^{-1}\left(L_{H, E}(t)\right)\right)$ \\
\hline TC-FTL-R & $\sqrt{ }$ & $Q_{Q-E}^{-1}\left(L_{H, E}(t)\right)$ \\
\hline TC-AHSE-R & $*$ & $\left(Q_{Q-E}^{-1}\left(L_{H, E}(t)\right), P_{\text {CHP.MAX }}\right)$ \\
\hline TC-FPG-R & $\sqrt{ }$ & $\begin{array}{ll}P_{\text {CHP.MAX }} & \\
& \end{array}$ \\
\hline
\end{tabular}

Among the 28 policies in Table 1, the operating strategies that are often discussed are TC-FTL-L, EC-FTL-L, EC-FEL-R, and TC-FEL-R, while almost none of the other operating strategies have been analyzed in detail.

According to the optimization theory, if a strategy does not satisfy the KKT condition, it is definitely not the optimal 
strategy. The KKT conditions of models $1-16$ are given in supplementary material S.1 in reference [23]. Testing the KKT conditions of models 1-16 for these strategies, it is shown that EC-AHPE-L, EC-FEL-L, EC-AHSE-L, EC-FPG-L, EC-AHSE$\mathrm{R}$, and EC-FPG-R that are labeled " $x$ " in Table 1 are impossible as optimal strategies (the proof that strategy EC-FEL-L does not satisfy the KKT condition is shown in supplementary material S.2 in reference [23], and other strategies can be proved according to similar method).

For the strategies that are labeled "*," the test that these strategies satisfy the KKT condition can be expressed as testing whether the real solution of the following algebraic equations is within the scope of their strategies (see supplementary material S.4 in reference [23] for the derivation process). That is, other 9 kinds of strategies that are labeled “*” in Table 1 may be an optimal strategy, only if a real number solution of the equation (23) is within the specified range.

$$
A+B \cdot f+C f^{2}+D f^{3}+E f^{4}=0
$$

where the parameters $A, B, C, D$, and $E$ can be obtained using power generation efficiency coefficients $a, b$, and $c$ and the parameter $d$ by equation (24).

$$
\left\{\begin{array}{l}
A=a^{2} \cdot d-a \\
B=2 \cdot a \cdot b \cdot d \\
C=2 \cdot a \cdot c \cdot d+c \\
D=2 \cdot c \cdot b \cdot d \\
E=c^{2} \cdot d
\end{array}\right.
$$

The parameter $d$ can be obtained by formula in Table 2 . Among them, strategies EC-IG-L, TC-IG-L, EC-IG-R, and TC-IG-R adopt the same formula to calculate parameter $d$. Strategies TC-AHSE-L and TC-AHSE-R use another formula, and so on.

If the real solution $f$ of equation (23) makes $P_{\mathrm{CHP}}(t)=$ $f \cdot P_{\text {CHP,MAX }}$ within the range given in Table 2 , then the corresponding strategy is the optimal strategy. The column RAG in Table 2 is the range of generate in the strategy. If the real solution $f$ of equation (23) makes $P_{\mathrm{CHP}}(t)=f \cdot P_{\mathrm{CHP}, \mathrm{MAX}}$ beyond the range, it means that the strategy does not meet the KKT condition.

Assume the cold, heat, and electricity loads are $L_{C}(t)$, $L_{H}(t)$, and $L_{E}(t)$ at time $t$, in order to determine whether EC-IG-L is the optimal strategy. The parameter $d$ is firstly obtained based on equation in Table 2 , and then the parameters $A, B, C, D$, and $E$ of equation (23) are obtained based on equation (24). If the real solution of equation (23) makes $P_{\mathrm{CHP}, \mathrm{MIN}}<f \cdot P_{\mathrm{CHP}, \mathrm{MAX}}<Q_{\mathrm{Q}-E}^{-1}\left(L_{H}(t)\right)$, the strategy EC-IG-L is the optimal strategy; otherwise, it is not the optimal strategy.

Testing KKT conditions, these strategies that are labeled " $\sqrt{ }$ " in Table 1 are most likely to be optimal strategies. The candidate optimal strategies that are set for electric cooling or thermal cooling consist of the strategies labeled “*” and " $\sqrt{ }$ " in Table 1 . It must be pointed out that the decision variables of each strategy can be obtained rapidly according to the load balance, so the operational cost of each strategy can be obtained rapidly.

At present, only FTL-L and FEL-R strategies have been widely studied, while other operational strategies have been almost ignored. In this paper, KKT conditions of the optimal operation model are used to comprehensively investigate all possible strategies, and the conditions for them to become the optimal strategies are obtained.

\subsection{The Candidate Optimal Operation Strategies for Both} Cooling Modes Working Simultaneously. It is shown in supplementary material S.3 in reference [23] that the KKT condition is not satisfied when two kinds of cooling devices work at same time except several special situations. It means that there are not almost optimal strategies when both cooling modes work simultaneously.

When the ECEL and TCEL are located on the both sides of the Q-E curve, there is an intersection of the line connecting ECEL and TCEL with Q-E curve, as the intersection WA in Figure 2. The intersection WA can be obtained to solve the equations (17) and (20), where $L_{\mathrm{EEL}}(t)=P_{\mathrm{CHP}}(t)$ and $L_{\mathrm{EHL}}(t)=Q_{\mathrm{CHP}}(t)$. The result is as follows:

$$
\begin{cases}P_{\mathrm{CHP}}(t)=\frac{\left(L_{H}-L_{H, E} / L_{E, E}-L_{E}(t)\right) \cdot L_{E}(t)-L_{H, E}}{\left(L_{H}-L_{H, E} / L_{E, E}-L_{E}(t)\right)-\left(1-\eta_{\mathrm{CHP}, E}\right) \cdot\left(\eta_{\mathrm{CHP}, H} / \eta_{\mathrm{CHP}, E}\right)}, & P_{\mathrm{CHPMAX}}>P_{\mathrm{CHP}}(t)>P_{\mathrm{CHPMIN}}, \\ Q_{\mathrm{CHP}}(t)=\frac{L_{H}-L_{H, E}}{L_{E, E}-L_{E}(t)} \cdot\left(P_{\mathrm{CHP}}(t)-L_{E}(t)\right)+L_{H, E}, & Q_{\mathrm{CHPMAX}}>Q_{\mathrm{CHP}}(t)>Q_{\mathrm{CHPMIN}} .\end{cases}
$$

The cold, heat, and electricity load will be supplied by the CCHP system without the boilers and purchasing/selling electricity from grid. The strategy is called the TEWM (the thermal electricity while meet). Four regions are defined by equation (27), and then the condition that there is intersection WA can be intuitively seen from Figure 2 that ECEL is located at $A 1$ or $A 3$, and TCEL is located at $A 3$ or $A 4$, that is, 
TABLE 2: The parameter $d$ and the range of the solution.

\begin{tabular}{lcr}
\hline Strategy & $d$ & RAG \\
\hline EC-IG-L & & $\left(P_{\mathrm{CHP}, \mathrm{MIN}}, Q_{\mathrm{Q}-E}^{-1}\left(L_{H}(t)\right)\right)$ \\
TC-IG-L & $\left(\rho_{\mathrm{GRID}}^{\text {Purchase }}-\eta_{\mathrm{CHP}, H} \times\left(\rho_{\mathrm{BL}} / \mathrm{LHV} \times \eta_{\mathrm{BL}}\right) /\left(\rho_{\mathrm{CHP}} / \mathrm{LHV}\right)-\eta_{\mathrm{CHP}, H} \cdot\left(\rho_{\mathrm{BL}} / \mathrm{LHV} \cdot \eta_{\mathrm{BL}}\right)\right)$ & $\left(P_{\mathrm{CHP}, \mathrm{MIN}}, Q_{\mathrm{Q}-E}\left(L_{H, E}(t)\right)\right)$ \\
$\begin{array}{l}\text { EC-IG-R } \\
\text { TC-IG-R }\end{array}$ & $\left(P_{\mathrm{CHP}, \mathrm{MIN}}, L_{E, E}(t)\right)$ & $\left(P_{\mathrm{CHP}, \mathrm{MIN}}, L_{E}(t)\right)$ \\
\hline TC-AHSE-L & $\left(\rho_{\mathrm{GRID}}^{\text {sell }} /\left(\rho_{\mathrm{CHP}} / \mathrm{LHV}\right)\right)$ & $\left(L_{E}(t), P_{\mathrm{CHP}, \mathrm{MAX}}\right)$ \\
TC-AHSE-R & $\left(\rho_{\mathrm{GRID}}^{\mathrm{Sell}}-\eta_{\mathrm{CHP}, H} \cdot\left(\rho_{\mathrm{BL}} / \mathrm{LHV} \cdot \eta_{\mathrm{BL}}\right) / \rho_{\mathrm{CHP}}-\eta_{\mathrm{CHP}, H} \cdot\left(\rho_{\mathrm{BL}} / \mathrm{LHV} \cdot \eta_{\mathrm{BL}}\right)\right)$ & $\left(Q_{Q-E}^{-1}\left(L_{H, E}(t)\right), P_{\mathrm{CHP}, \mathrm{MAX}}\right)$ \\
\hline EC-BWSE-R & $\left(\rho_{\mathrm{GRID}}^{\mathrm{Purchase}} /\left(\rho_{\mathrm{CHP}} / \mathrm{LHV}\right)\right)$ & $\left(L_{E, E}(t), Q_{\mathrm{Q}-E}^{-1}\left(L_{H}(t)\right)\right)$ \\
TC-BWSE-R & & $\left(L_{E}(t), Q_{Q-E}^{-1}\left(L_{H, E}(t)\right)\right)$ \\
\hline TC-AHPE-L & & $\left(Q_{Q-E}^{-1}\left(L_{H, E}(t)\right), L_{E}(t)\right)$ \\
\hline
\end{tabular}

$$
\begin{aligned}
& S_{\mathrm{TEWM}}=\left[\begin{array}{c}
(\mathrm{ECEL} \in A 1 . \text { and.TCEL } \in A 2) \\
\text {.or. }(\mathrm{ECEL} \in A 1 \text {.and.TCEL } \in A 4) \\
\text {.or. }(\mathrm{ECEL} \in A 3 \text {.and.TCEL } \in A 2) \\
\text {.or. }\left(\mathrm{ECEL} \in \text { A3.and.TCEL } \in A 4 \text {.and. } Y \in\left(L_{E}(t), P_{\mathrm{CHP}, \mathrm{MAX}}\right)\right)
\end{array}\right] \text {, } \\
& \left\{\begin{array}{l}
A 1=\left(L_{H}(t) \leq Q_{\mathrm{Q}-E}\left(L_{E, E}(t)\right) \cdot \text { and } L_{E, E}(t) \in\left(P_{\mathrm{CHP}, \mathrm{MIN}}, P_{\mathrm{CHP}, \mathrm{MAX}}\right)\right), \\
A 2=\left(L_{H, E}(t)>Q_{\mathrm{Q}-E}\left(L_{E}(t)\right) \cdot \text { and } L_{E}(t) \in\left(P_{\mathrm{CHP}, \mathrm{MIN}}, P_{\mathrm{CHP}, \mathrm{MAX}}\right)\right), \\
A 3=\left(L_{H}(t) \leq Q_{\mathrm{CHP}, \mathrm{MAX}} \cdot \text { and } L_{E, E}(t) \in\left(P_{\mathrm{CHP}, \mathrm{MAX}}, \infty\right)\right), \\
A 4=\left(L_{H, E}(t)>Q_{\mathrm{CHP}, \mathrm{MAX}} \cdot \text { and } L_{E}(t) \in\left(P_{\mathrm{CHP}, \mathrm{MIN}}, P_{\mathrm{CHP}, \mathrm{MAX}}\right)\right),
\end{array}\right. \\
& Y=\left(Q_{\mathrm{CHP}, \mathrm{MAX}}-L_{H, E}\right) \times \frac{L_{E, E}-L_{E}}{L_{H}-L_{H, E}}+L_{E} .
\end{aligned}
$$

When ECEL is located at $A 3$ and TCEL is located at $A 4$, an additional condition is need. The intersection $Y$ of the line connecting ECEL and TCEL with maximum heat recovery line should be less than the maximum power generation. By substituting $L_{\mathrm{EHL}}(t)=Q_{\mathrm{CHP}, \mathrm{MAX}}$ in equation (20), the intersection $Y$ can be expressed as equation (28).

Using equations (3), (4), and (25), the output of the electric chiller and absorption chiller is

$$
\left\{\begin{array}{l}
Q_{\mathrm{EC}}(t)=\left(P_{\mathrm{CHP}}(t)-L_{E}(t)\right) \cdot \mathrm{COP}_{\mathrm{EC}}, \\
Q_{\mathrm{ABC}}(t)=\left(Q_{\mathrm{CHP}}(t)-L_{H}(t)\right) \cdot \mathrm{COP}_{\mathrm{EC}} .
\end{array}\right.
$$

When the line connecting ECEL and TCEL intersects the maximal heat recovery, like WE1 and WD1 in Figure 2, the operation strategies for the equivalent load of the WE1 and WD1 may be optimal.

The condition that there is WE1 is equation (30), in which $Y$ is calculated by equation (28).

$$
L_{E, E}(t) \leq Y \leq L_{E}(t) \text {.and. } Y \leq P_{\mathrm{CHP}, \mathrm{MAX}} .
$$

The strategy to meet the equivalent load WE1 is called SE-FPG (selling electricity and full power generation). Using SE-FPG, the generator works at full power, the power generation is larger than $Y$, the excess power will be sold to the grid, and the heat recovery is equal to the equivalent heat load.
The condition that there is WD1 is equation (31), in which $Y$ is obtained by equation (28).

$$
L_{E, E}(t) \leq Y \leq L_{E}(t) \text {.and. } Y>P_{\mathrm{CHP}, \mathrm{MAX}} .
$$

PE-FPG (purchasing electricity and full power generation) is used to meet the WD1. In this case, the generator's full power is generated, but because $Y>P_{\mathrm{CHP}, \mathrm{MAX}}$, the electricity energy is purchased from grid to meet the equivalent electricity load.

If equation (32) is true, the line connecting ECEL and TCEL intersects the maximal generation, like WD2 in Figure 2. BW-FPG (boiler working and full power generation) is used to meet the equivalent load.

$$
\left\{\begin{array}{l}
Z=\left(P_{\mathrm{CHP}, \mathrm{MAX}}-L_{E}(t)\right) \times \frac{L_{H}(t)-L_{H, E}(t)}{L_{E, E}(t)-L_{E}(t)}+L_{H, E}(t), \\
L_{H}(t) \leq Z \leq L_{H, E}(t) \text {.and. } Z>Q_{\mathrm{CHP}, \mathrm{MAX}} .
\end{array}\right.
$$

Because $Z \geq Q_{\mathrm{CHP}, \mathrm{MAX}}$, when the generator works in its maximal capacity, the boiler still needs to be turned on to provide heat, but the purchasing/selling electricity is zero.

When equation (33) is true, the intersection of the minimal heat recovery and the line connecting ECEL and TCEL may occur, like WF1 in Figure 2. 


$$
\left\{\begin{array}{l}
Z=\left(Q_{\mathrm{CHP}, \mathrm{MIN}}-L_{H, E}(t)\right) \times \frac{L_{E, E}(t)-L_{E}(t)}{L_{H}(t)-L_{H, E}(t)}+L_{E}(t), \\
L_{E}(t) \leq Z \leq L_{E, E}(t) \text {.and. } Z>P_{\mathrm{CHP}, \mathrm{MIN}} .
\end{array}\right.
$$

PE-MPG (purchasing electricity and minimal power generation) may be an optimal strategy to meet the load WF1. The generator works at its minimal output, and the electricity is purchased from grid.

BW-MPG (boiler working and minimal power generation) is a strategy at the intersection point of the minimal generation line and the line connecting ECEL and TCEL, like WF2 in Figure 2. The equivalent heat loaded in the point can be calculated like equation (27). Using BW-MPG, the generator works in the minimal output and the boiler works to supply thermal.

$$
\left\{\begin{array}{l}
Y=\left(P_{\mathrm{CHP}, \mathrm{MIN}}-L_{E}(t)\right) \times \frac{L_{H}(t)-L_{H, E}(t)}{L_{E, E}(t)-L_{E}(t)}+L_{H, E}(t), \\
L_{H}(t) \leq Y \leq L_{H, E}(t) . \text { and. } Y>Q_{\mathrm{CHP}, \mathrm{MIN}} .
\end{array}\right.
$$

The candidate optimal operation strategies' cooling modes working simultaneously and their conditions are shown in Table 3.

Finally, the separation pattern (SP) as a strategy means that the generator is off, the cold load is met by electric chiller, the boiler product heat energy meets the heat load, and the electricity is purchased from grid to supply the electricity load. If the cost using SP is the minimum, the SP will be the operation strategy.

\section{Simulations Based on the Candidate Optimal Operation Strategies}

Totally, 28 kinds of operation strategies may be the optimal strategies. But ECEL is either on the left side of the border or on the right side of the border, not simultaneously on both sides. And the candidate optimal strategies' cooling modes working simultaneously have their conditions. As a result, the candidate optimal strategies for a load maybe include only 10 kinds of operation strategies. Moreover, the decision variables and operation cost can be obtained quickly.

The basic idea about the chronological simulation of CCHP is calculating the cost for the load using each candidate optimal strategy and selecting the strategy whose cost is minimum as the optimal strategy.

Firstly, the equivalent loads of ECEL and TCEL are calculated according to equations (18) and (19). Then, according to the ECEL or TCEL located on the left or the right side of the boundary to select the strategies in Table 1 , the decision variables and the operation cost for each selected strategy are calculated.

Thirdly, the strategies that meet the condition in Table 3 are selected, and the decision variables and operation cost are calculated.
TABLE 3: The candidate optimal operation strategies' cooling modes working simultaneously.

\begin{tabular}{ll}
\hline Strategy & Condition \\
\hline TEWM & Equation (26) \\
PE-FPG & Equation (30) \\
SE-FPG & Equation (31) \\
BW-FPG & Equation (32) \\
PE-MPG & Equation (33) \\
BE-MPG & Equation (34) \\
\hline
\end{tabular}

Selecting the strategy with minimal cost as the optimal, the decision variables and cost of the optimal strategy are coded, as shown in Figure 3.

\section{Case Study}

A CCHP system that supplies energy for a hospital load is used as a case to test the present algorithm. The maximum cold load is $2579.6 \mathrm{~kW}$, the maximum heat load is $2879.7 \mathrm{~kW}$, and the maximum electricity load is $1000 \mathrm{~kW}$.

The generator capacity is $900 \mathrm{~kW}$, and the parameters of the CCHP and the prices of the purchasing electricity and gas are shown in Table 4 . The price of selling electricity to grid is $0.5 ¥ / \mathrm{kWh}$, which is higher than coal unit selling price at about $0.1 ¥ / \mathrm{kWh}$.

For convenience, three states are defined according to ECEL and TCEL. If ECEL and TCEL are, respectively, located on both sides of the boundary, it is called state 1 . When ECEL and TCEL are located on the left side of the border, it is known as state 2. It is called state 3 , when ECEL and TCEL are on the right side of the border. In this case, the number of state 1 , state 2 , and state 3 is 2227,6341 , and 192 separately.

The strategies selected as optimal strategies are shown in Table 5 . In state 1 , it shows that there are 778 hours when TEWM is the optimal strategy. There are 271 hours when TC-FTL- $R$ is the optimal strategy. Selling electricity to grid in TC-FTL-R can reduce its cost; if the sales income is greater than the generation cost, the strategy could be the strategy with the minimal cost.

The scatter plot of the ECELs and TCELs in state 1 are shown in Figure 4(a). ECELs are blue dots, and the TCELs are black points. They are equivalent loads, when TC-FTL-R is the optimal strategy. The yellow dots represent the ECELs and the claret-colored points represent the TCELs. They are the equivalent loads, when TEWM is the optimal strategy. In Figure 4(a), it shows that, for the optimal strategy of PE-FPG in state 1 (Table 5), the equivalent electricity loads of 1178 points for ECELs and TCELs are higher than the maximal power generation, where the red points represent the ECELs and the green points represent the TCELs.

The ECELs and TCELs are all on the left side of the boundary in state 2 . The ECELs and TCELs are shown in Figure 4(b). When TC-FEL-L is the optimal strategy in 5711 hours, the equivalent loads are shown as blue points/black dots as ECELs/TCELs. The traditional strategy in this case is FTL. If power generation cost of the CCHP is less than the purchasing electricity price, the cost using FEL is less than that using FTL, so TC-FEL-L is an optimal strategy in most 


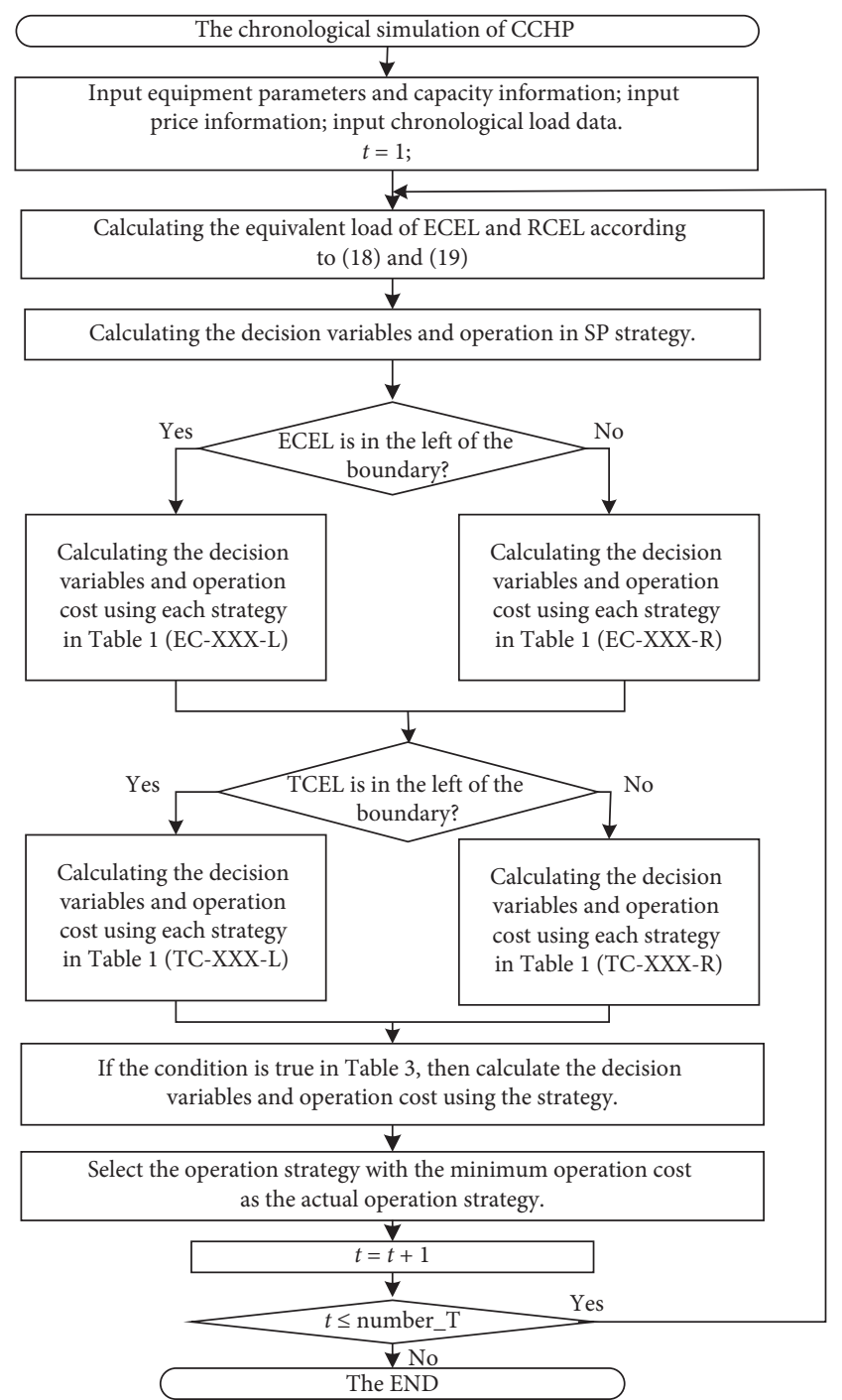

FIgURE 3: The chronological simulation flow diagram.

TABLE 4: The parameters and price.

\begin{tabular}{lc}
\hline Item & Value \\
\hline Generator & $\begin{array}{c}\text { Maximum capacity: } 900 \mathrm{~kW} \text {, the } \\
\text { generation efficiency parameter: } a=-0.2, \\
b=0.4, c=0.1 \text {. The waste heat recovery } \\
\text { efficiency CHP, } H=0.8 \\
\text { Maximum capacity: } 1500 \mathrm{~kW} \text {, the } \\
\text { coefficient of energy efficiency } \\
\text { COPABC }=0.8 .\end{array}$ \\
$\begin{array}{l}\text { Absorption chiller } \\
\text { Maximum capacity: } 1200 \mathrm{~kW} \text {, the } \\
\text { coefficient of energy efficiency } \\
\text { COPEC }=4 .\end{array}$ \\
$\begin{array}{l}\text { Electric chiller } \\
\text { Boiler }\end{array}$ \\
$\begin{array}{l}\text { Gas lower heating } \\
\text { value, LHV }\end{array}$ \\
$\begin{array}{l}\text { The gas price } \\
\text { Selling electricity } \\
\text { price } \\
\text { Purchasing electricity } \\
\text { price }\end{array}$
\end{tabular}

TABLE 5: The optimal strategy.

\begin{tabular}{|c|c|c|}
\hline State 1 & Strategy & Num. \\
\hline \multirow{16}{*}{ State 1} & SP & 0 \\
\hline & TEWM & 778 \\
\hline & EC-IG-L & 0 \\
\hline & EC-FTL-L & 0 \\
\hline & TC-IG-R & 0 \\
\hline & TC-FEL-R & 0 \\
\hline & TC-BWSE-R & 0 \\
\hline & TC-FTL-R & 271 \\
\hline & TC-AHSE-R & 0 \\
\hline & TC-FPG-R & 0 \\
\hline & SE-FPG & 0 \\
\hline & PE-FPG & 1178 \\
\hline & BW-FPG & 0 \\
\hline & BW-MPG & 0 \\
\hline & PE-MPG & 0 \\
\hline & Summation & 2227 \\
\hline \multirow{15}{*}{ State 2} & $\mathrm{SP}$ & 630 \\
\hline & EC-IG-L & 0 \\
\hline & TC-IG-L & 0 \\
\hline & TC-FTL-L & 0 \\
\hline & TC-AHPE-L & 0 \\
\hline & TC-FEL-L & 5711 \\
\hline & TC-AHSE-L & 0 \\
\hline & TC-FPG-L & 0 \\
\hline & PE-FPG & 0 \\
\hline & PE-MPG & 0 \\
\hline & - & 0 \\
\hline & - & 0 \\
\hline & - & 0 \\
\hline & - & 0 \\
\hline & Summation & 6341 \\
\hline \multirow{16}{*}{ State 3} & $\mathrm{SP}$ & 0 \\
\hline & EC-IG-R & 0 \\
\hline & EC-FEL-R & 171 \\
\hline & EC-BWSE-R & 0 \\
\hline & EC-FTL-R & 21 \\
\hline & TC-IG-R & 0 \\
\hline & TC-FEL-R & 0 \\
\hline & TC-BWSE-R & 0 \\
\hline & TC-FTL-R & 0 \\
\hline & TC-AHSE-R & 0 \\
\hline & TC-FPG-R & 0 \\
\hline & BW-FPG & 0 \\
\hline & SE-FPG & 0 \\
\hline & BW-MPF & 0 \\
\hline & - & 0 \\
\hline & Summation & 192 \\
\hline
\end{tabular}

hours. When the equivalent loads are very small, as shown by yellow dots and claret-colored points in Figure 4(b), the operation costs supplying energy by CCHP are higher than those by separation pattern. Furthermore, as shown in Table 5 , in state 2, there are 630 hours when SP is the optimal strategy.

As for state 3, as shown in Table 5, there are 171 hours when EC-FEL-R is the optimal strategy and 21 hours when EC-FTL-R is the optimal strategy. In state 3, ECELs and TCELs are on the right side of boundary as shown in Figure 4(b). EC-FTL-R is the optimal strategy when the 


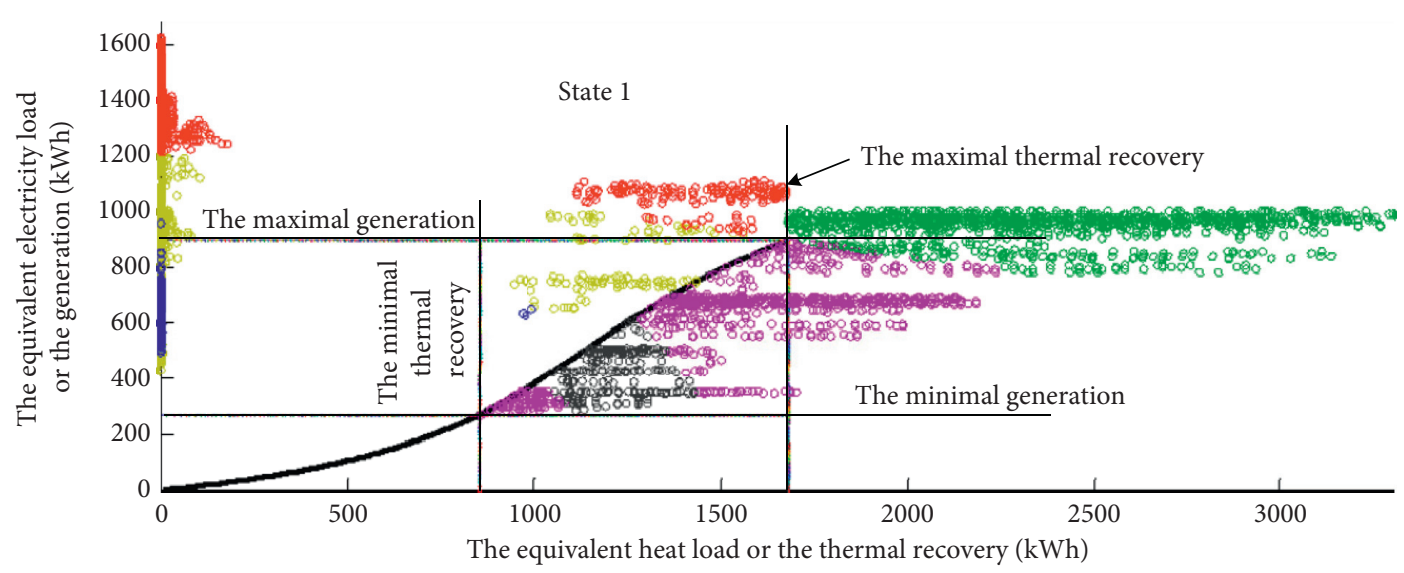

(a)

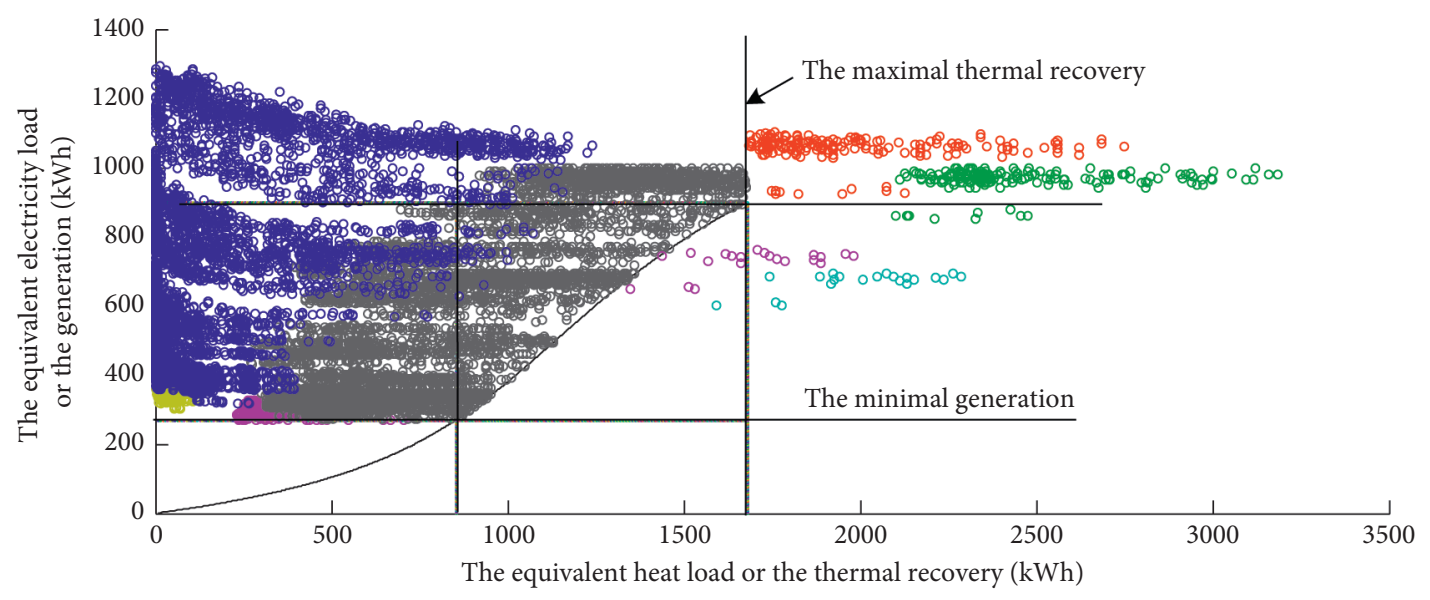

(b)

Figure 4: The scatterplots of the ECELs and TCELs. (a) ECEL and TCEL scatterplot in state 1. (b) ECEL and TCEL scatterplot in state 2 and state 3.

TABLE 6: The results of simulation.

\begin{tabular}{lcr}
\hline Item & The presented method & HET \\
\hline CCHP generation (kWh) & $5,164,040.76$ & $3,075,922.70$ \\
CCHP thermal recovery (kWh) & $10,724,344.68$ & $6,352,979.46$ \\
Selling electricity (kWh) & $45,080.84$ & 0.00 \\
Purchasing electricity (kWh) & $521,867.97$ & $2,663,615.86$ \\
Supplying heat by boiler (kWh) & $81,639.37$ & $1,630,075.48$ \\
Wasted heat (kWh) & $2,296,472.99$ & 0.00 \\
Cooling by electric chiller (kWh) & $954,267.86$ & $1,349,110.55$ \\
Cooling by absorption chiller (kWh) & $4,980,597.90$ & $4,585,755.21$ \\
Operation cost (¥) & $5,063,864.42$ & $6,013,709.08$ \\
\hline
\end{tabular}

equivalent electricity loads are less than the maximal power generation. Otherwise, the EC-FEL-R is the optimal strategy.

The results of the annual simulation based on the presented method and the HET rules are shown in Table 6. Because the presented method uses the optimal strategy, the annual operation cost using the presented method decreases by $20.69 \%$ than that of HET. This means the generator can produce more electricity and the purchasing electricity from grid can be reduced with presented method, even if some heat is wasted, the operation cost saving can also be achieved.

A primal dual algorithm [20] is used to solve the optimal models 1-16 for several typical loads. The results of the optimal problem are consistent with the results of the presented method. One of the results is shown in Table 7. The results of two methods have the same characteristics that the cold load, heat load, and the electricity load are met by co-generation units, that is, the boiler does not work and the selling/purchasing electricity to/from grid is zero. The results 
TABLE 7: Results of the solution of the optimal model.

\begin{tabular}{lcc}
\hline Item & The presented method & The primal dual optimization algorithm \\
\hline Cold load & 392.2 & 392.28 \\
Heat load & 1104.5 & 1104.5 \\
Electricity load & 867.1 & 867.12 \\
Power generation $(\mathrm{kWh})$ & 868.1 & 868.48 \\
Purchasing electricity from grid (kWh) & 0 & 0 \\
Selling electricity to grid (kWh) & 0 & 0 \\
Producing heat energy by boiler (kWh) & 0 & 0 \\
Cooling by electric chiller (kWh) & 3.96 & 4.15 \\
Cooling by absorption chiller $(\mathrm{kWh})$ & 388.3 & 388.63 \\
Operation cost & 1172.00 & 1172.54 \\
\hline
\end{tabular}

display an interesting fact that the decision variables obtained according to the optimal strategy in the presented method do not iterate, so the results are more precise than those of an optimal algorithm.

This simulation algorithm is implemented on MATLAB platform. Annual simulation takes about 3.5 seconds. The most time-consuming work in the simulation is solving algebraic equation (23). The time consumption by HET based simulation is about 0.58 seconds.

\section{Conclusion}

In this paper, all kinds of operation strategies of the CCHP with two cooling methods are investigated according to the KKT conditions of the optimal operation model. Among all the feasible operation strategies, most of them do not meet the KKT condition, and only 43 strategies may be the optimal ones. These strategies constitute the candidate set. The conditions of the optimal operation strategies in the candidate set are obtained, and it is easy to calculate, so the optimal operation strategies can be easily obtained from the candidate set.

KKT condition analysis based on the optimal operation model shows that when both cooling modes work simultaneously, only TEWM, PE-FPG, PE-MPG, BW-FPG, PE-MPG, and BE-MPG are possibly optimal, while other combinations strategies are not optimal. When the cold demand is met by electric chiller or absorption chiller alone, traditional FTL-L and FEL-R may be the optimal strategies in some cases, but there are also other strategies that cannot be ignored in the case of electricity price and load, such as FTL-R and FEL-L and so on.

A rule based on CCHP simulation method that selects the strategy with minimal cost like the optimal strategy during the candidate strategies is developed. A CCHP system that supplies energy for a hospital load is used as a case to test the present algorithm. Compared with the traditional simulation based on HET operation strategy, the results show that the operation cost of the proposed method is less than that of the HET strategy. Compared with the results of the optimization model, the cost of the strategy in this paper is consistent with the optimization result at the selected typical moments, which shows that the method in this paper is based on the optimization strategy. The calculation time of this method is a little longer than that of HET simulation, but it is still fast enough (it takes 3.5 seconds to simulate 8760 hours). The presented method has small time consumption but can simulate the optimal operation. The proposed approach can be used as a tool to estimate the operation cost in investment planning algorithm. The storage devices and renewable energy will be considered using the proposed method in subsequent work.

\section{Nomenclature}

Symbol

$a, b, c$ : The coefficient of power generation cost function

AHPE: Abandoning heat and purchasing electricity strategy

AHSE: Abandoning heat and selling electricity strategy

BWSE: Boiler working and selling electricity strategy

BW-: Boiler working, prefixion

CCHP: Combined cooling, heat, and power (CCHP) system

COP: Coefficient of performance

EC-: Electric cooling, prefixion

ECEL: The electric cooling equivalent load

FEL: $\quad$ Following electricity load strategy

FPG: $\quad$ Full power generation strategy

FTL: Following thermal load strategy

IG: Insufficient generating, operation strategy

LHV: Low heat value

MPG: Minimal power generation strategy

PE-: $\quad$ Purchasing electricity, prefixion

PGU: Power generation unit

TC-: Thermal cooling, prefixion

TCEL: Thermal cooling equivalent load strategy

TEWM: Thermal electricity while meet strategy

SE-: $\quad$ Selling electricity, prefixion

SP: $\quad$ Separate supply energy model

-L: $\quad$ Locate on the left of boundary, suffix

-R: $\quad$ Located on the right of boundary, suffix

Variables

$L:$ The load (kWh)

$P$ : The electric energy ( $\mathrm{kWh})$

$Q$ : The heat or cold energy $(\mathrm{kWh})$

$t$ : Time variable

$x$ : Binary variable, $x=0$, selling electricity model; $x=1$, purchasing electricity model

$Y$ : The equivalent electricity energy for electric chiller and absorption chiller 
$Z$ : The equivalent thermal energy for electric chiller and absorption chiller

Subscript

ABC: Absorption chiller

BL: $\quad$ Boiler

C: $\quad$ Cold load

CHP: Combined heat and power system

E: $\quad$ Electricity load

EC: Electric chiller

E,E: The equivalent electricity load when electric chiller supplies the cooling service

EEL: The equivalent electricity load while the electric chiller and the absorption chiller work simultaneously

EHL: The equivalent heat load while the electric chiller and the absorption chiller work simultaneously

GRID: The purchasing or selling electricity from/to grid

$H: \quad$ Heat

$H, E$ : The equivalent heat load when absorption chiller supplies the cooling service

MAX: The maximal value

MIN: The minimal value

Superscript

Purchase: Purchasing electricity from grid

Sell: $\quad$ Selling electricity to grid.

\section{Data Availability}

The data used to support the findings of this study are included within the article.

\section{Conflicts of Interest}

The authors declare that there are no conflicts of interest regarding the publication of this paper.

\section{Acknowledgments}

This study was funded by the National Natural Science Foundation of China (Project no. 61876105).

\section{Supplementary Materials}

The supplementary materials in this work include "S.1. The Lagrangian function and KKT condition of models 1-16," "S.2. Testing the KKT conditions for the EC-FEL-L," "S.3. Testing the KKT condition for the strategies of both cooling modes working simultaneously," and "S.4. Obtaining equation (23)." The detailed supplementary materials are presented in reference [24]. (Supplementary Materials)

\section{References}

[1] U.S.Department of Energy \& United States Environmental Protection Agency, Combined Heat and Power: Evaluating the Benefits of Greater Global Investment, International Energy Agency, Paris, France, 2008.

[2] International Energy Agency (IEA), Combined Heat and Power: A Clean Energy Solution, Environmental Protection Agency, Washington, DC, USA, 2012.
[3] M. H. Ahmadi, M. Alhuyi Nazari, M. Sadeghzadeh et al., "Thermodynamic and economic analysis of performance evaluation of all the thermal power plants: a review," Energy Science \& Engineering, vol. 7, no. 1, pp. 30-65, 2019.

[4] H. Cho, A. D. Smith, and P. Mago, "Combined cooling, heating and power: a review of performance improvement and optimization," Applied Energy, vol. 136, pp. 168-185, 2014.

[5] W. Gu, Z. Wu, R. Bo et al., "Modeling, planning and optimal energy management of combined cooling, heating and power microgrid: a review," International Journal of Electrical Power \& Energy Systems, vol. 54, pp. 26-37, 2014.

[6] P. J. Mago, N. Fumo, and L. M. Chamra, "Performance analysis of CCHP and CHP systems operating following the thermal and electric load," International Journal of Energy Research, vol. 33, no. 9, pp. 852-864, 2009.

[7] A. Moran, P. J. Mago, and L. M. Comra, "Thermo economic modeling of micro-CHP for small commercial applications," International Journal of Energy Research, vol. 32, no. 9, pp. 808-823, 2010.

[8] J. Wang, J. Sui, and H. Jin, “An improved operation strategy of combined cooling heating and power system following electrical load," Energy, vol. 85, pp. 654-666, 2015.

[9] J. Zhang, S. Cao, L. Yu, and Y. Zhou, "Comparison of combined cooling, heating and power (CCHP) systems with different cooling modes based on energetic, environmental and economic criteria," Energy Conversion and Management, vol. 160, pp. 60-73, 2018.

[10] H. Ahn, J. D. Freihaut, and D. Rim, "Economic feasibility of combined cooling, heating, and power (CCHP) systems considering electricity standby tariffs," Energy, vol. 169, pp. 420-432, 2019.

[11] M. Liu, Y. Shi, and F. Fang, "A new operation strategy for CCHP systems with hybrid chillers," Applied Energy, vol. 95, pp. 164-173, 2012.

[12] C. Y. Zheng, J. Y. Wu, and X. Q. Zhai, "A novel operation strategy for CCHP systems based on minimum distance," Applied Energy, vol. 128, pp. 325-335, 2014.

[13] F. Ren, J. Wang, S. Zhu, and Y. Chen, "Multi-objective optimization of combined cooling, heating and power system integrated with solar and geothermal energies," Energy Conversion and Management, vol. 197, Article ID 111866, 2019.

[14] D. E. Ghersi, M. Amoura, K. Loubar, U. Desideri, and M. Tazerout, "Multi-objective optimization of CCHP system with hybrid chiller under new electric load following operation strategy," Energy, vol. 219, Article ID 119574, 2021.

[15] Z. Han and S. Guo, "Investigation of operation strategy of combined cooling, heating and power (CCHP) system based on advanced adiabatic compressed air energy storage," Energy, vol. 160, pp. 290-308, 2018.

[16] L. Gao, Y. Hwang, T. Cao et al., "An overview of optimization technologies applied in combined cooling, heating and power systems," Renewable and Sustainable Energy Reviews, vol. 114, Article ID 109344, 2019.

[17] Y. Cao, Q. Wang, J. Du, S. Nojavan, K. Jermsittiparsert, and N. Ghadimi, "Optimal operation of CCHP and renewable generation-based energy hub considering environmental perspective: an epsilon constraint and fuzzy methods," Sustainable Energy, Grids and Networks, vol. 20, Article ID 100274, 2019.

[18] A. Rong and R. Lahdelma, "An efficient envelope-based branch and bound algorithm for non-convex combined heat 
and power production planning," European Journal of Operational Research, vol. 183, no. 1, pp. 412-431, 2007.

[19] A. Sashirekha, J. Pasupuleti, N. H. Moin, and C. S. Tan, "Combined heat and power (CHP) economic dispatch solved using Lagrangian relaxation with surrogate subgradient multiplier updates," International Journal of Electrical Power \& Energy Systems, vol. 44, no. 1, pp. 421-430, 2013.

[20] B. Mohammadi-Ivatloo, M. Moradi-Dalvand, and A. Rabiee, "Combined heat and power economic dispatch problem solution using particle swarm optimization with time varying acceleration coefficients," Electric Power Systems Research, vol. 95, no. 1, pp. 9-18, 2013.

[21] Z. Xu, X. Guan, Q.-S. Jia, J. Wu, D. Wang, and S. Chen, "Performance analysis and comparison on energy storage devices for smart building energy management," IEEE Transactions on Smart Grid, vol. 3, no. 4, pp. 2136-2147, 2012.

[22] A. Bischi, L. Taccari, E. Martelli et al., "A detailed MILP optimization model for combined cooling, heat and power system operation planning," Energy, vol. 74, pp. 12-26, 2014.

[23] L. Guo, W. Liu, J. Cai, B. Hong, and C. Wang, "A two-stage optimal planning and design method for combined cooling, heat and power microgrid system," Energy Conversion and Management, vol. 74, pp. 433-445, 2013.

[24] Y. Li, B. Zou, F. Zhu, and J. Fu, "An optimal planning method for CCHP systems based on operation simulation," in Proceedings of the 2018 IEEE International Conference on Smart Energy Grid Engineering (SEGE)https://ieeexplore.ieee.org/ document/8499483, Oshawa, Canada, August 2018. 